\title{
Cerebral angioplasty using the Scepter XC dual lumen balloon for the treatment of vasospasm following intracranial aneurysm rupture
}

\author{
Jeremy J Heit, ${ }^{1}$ Omar Choudhri, ${ }^{2}$ Michael P Marks, ${ }^{1,2}$ Robert L Dodd, ${ }^{1,2}$ Huy M Do ${ }^{1,2}$
}

${ }^{1}$ Interventional Neuroradiology Division, Department of Radiology, Stanford University Hospital, Stanford, California, USA

${ }^{2}$ Department of Neurosurgery, Stanford University Hospital, Stanford, California, USA

\section{Correspondence to} Dr H M Do, Department of Radiology, Interventional Neuroradiology Division, Stanford University Hospital, 300 Pasteur Drive, S0047, Stanford, CA 94305, USA; huymdo@stanford.edu

Received 21 November 2013 Revised 11 December 2013 Accepted 12 December 2013 Published Online First 2 January 2014

\section{CrossMark}

\section{To cite: Heit JJ,}

Choudhri O, Marks MP,

et al. I Neurolntervent Surg 2015;7:56-61.

\section{ABSTRACT}

Background Cerebral vasospasm following subarachnoid hemorrhage (SAH) results in significant morbidity and mortality. Intra-arterial administration of calcium channel blockers or intracranial angioplasty may be performed when non-invasive medical management fails to prevent neurologic deterioration. Technical improvements in balloon catheters are expected to improve the success and safety of cerebral angioplasty. Objective To describe our initial experience with the new Scepter XC balloon catheter in cerebral vasospasm treatment following $\mathrm{SAH}$.

Design All patients who underwent cerebral angioplasty using the Scepter XC balloon for the treatment of medically refractory cerebral vasospasm after SAH were identified. Patient demographic information, procedural details, and outcome were obtained from electronic medical records.

Results Five consecutive patients undergoing vasospasm treatment with cerebral angioplasty using the Scepter XC were identified. All treated patients had medically refractory vasospasm that was moderate or severe. Angioplasty of the supraclinoid internal carotid artery, the A1 and A2 segments of the anterior cerebral artery, the $M 1$ and $M 2$ segments of the middle cerebral artery, the V4 segment of the vertebral artery, and the basilar artery was performed. All angioplasty procedures were technically successful, and the degree of vasospasm improved significantly following angioplasty. There were no complications related to the cerebral angioplasty procedures.

Conclusions The Scepter XC balloon catheter is safe and effective in the treatment of cerebral vasospasm following $\mathrm{SAH}$. The excellent trackability and stability of the balloon catheter and the extra compliant design of the balloon represent technical advancements in the endovascular armamentarium in the treatment of cerebral vasospasm.

\section{INTRODUCTION}

Cerebral vasospasm affects approximately twothirds of patients who present with aneurysmal subarachnoid hemorrhage (SAH). ${ }^{1}{ }^{2}$ Among these patients, up to one-third develop significant morbidity or even death. ${ }^{1-3}$ Consequently, significant effort and resources are harnessed to identify and prevent early signs of clinically significant vasospasm in the days following SAH.

Non-invasive treatment of cerebral vasospasm after aneurysmal SAH includes intravascular volume expansion with intravenous fluids, vasopressor administration to increase mean arterial pressure, and hemodilution. ${ }^{45}$ However, these measures to prevent cerebral vasospasm are insufficient in approximately $20 \%$ of patients, and patients may require more aggressive endovascular therapy in order to prevent vasospasm mediated cerebral infarction. ${ }^{6}$

There are two techniques available to neurointerventionalists in the treatment of cerebral vasospasm: (1) intra-arterial (IA) administration of arterial vasodilators, such as nicardipine, verapamil, or nimodipine, directly into the spastic vessel or (2) balloon angioplasty of the spastic vessel. ${ }^{7-10}$ Both treatment strategies are often employed, although angioplasty has been shown to offer more durable results. ${ }^{11-14}$ However, cerebral angioplasty carries a higher risk to the patient, including arterial dissection and vessel rupture, which may be catastrophic. ${ }^{15} 16$

Further advances in neuroendovascular devices, including more trackable guide catheters and angioplasty balloon catheters, extra compliant angioplasty balloons, and increased stability of angioplasty balloon catheters, are expected to result in a continued improvement in the safety profile of cerebral angioplasty for the treatment of vasospasm following aneurysmal SAH.

Here we describe our experience at a single center with the new coaxial dual lumen Scepter XC balloon (MicroVention Inc, Tustin, California, USA) in the treatment of cerebral vasospasm.

\section{MATERIALS AND METHODS}

The study was approved by our hospital's institutional review board and complied with the Health Insurance Portability and Accountability Act. We performed a retrospective review of our internal database and identified all patients undergoing endovascular vasospasm treatment by angioplasty using the Scepter XC balloon from July 2012 to September 2013. Patient demographic information and outcomes were obtained by review of our electronic medical records system.

All procedures were performed in a dedicated neuroangiography suite with a Siemens Artis Zee biplane system. The procedures were performed under general anesthesia with complete paralysis of the patient during the angioplasty portions of the intervention.

The common femoral artery was accessed with a 6 F Britetip vascular sheath (Cordis, Bridgewater, New Jersey, USA) or a Shuttle SL sheath (Cook Medical, Bloomington, Indiana, USA). Initial diagnostic cerebral angiograms were performed with either a $5 \mathrm{~F}$ VER catheter or a $5 \mathrm{~F}$ Berenstein II 
catheter positioned in the internal carotid artery (ICA) or the vertebral artery. Prior to vasospasm treatment, a guide catheter was positioned in the ICA or vertebral artery proximal to the affected vessels. IA nicardipine was infused at a rate of 15$30 \mathrm{mg} / \mathrm{h}$ for a period of $30-180 \mathrm{~min}$. Angioplasty with the Scepter XC balloon was performed in patients with moderate or severe vasospasm.

Prior to performing cerebral angioplasty, intravenous heparin was administered to achieve an activated clotting time of 250300 s. The Scepter XC balloon was prepared according to the manufacturer's instructions. The Scepter XC balloon was then advanced over a 0.014 inch Traxcess microwire (MicroVention, Tustin, California, USA) or a 0.014 inch Synchro 2 microwire (Boston Scientific, Natick, Massachusetts, USA) into the affected vessel. Angioplasty was carefully performed under continuous fluoroscopy with roadmap guidance, with slow and gentle inflation of the Scepter XC balloon.

The timing and duration of IA nicardipine therapy and angioplasty were at the discretion of the attending physician. In most cases, a total of $10-30 \mathrm{mg}$ of IA nicardipine were administered prior to cerebral angioplasty, and a total of $60 \mathrm{mg}$ of IA nicardipine were administered in a single case. The patient's blood pressure was continually monitored by an arterial line in order to maintain mean arterial pressure $>90$. Typically, IA nicardipine was administered while the Scepter XC balloon was prepared on the back table and, in most cases, IA nicardipine was also administered concomitantly with cerebral angioplasty.

In cases where IA nicardipine was administered concomitantly with angioplasty, the drug was administered through the guide catheter in either the ICA or vertebral artery. Next, the Scepter $\mathrm{XC}$ balloon was advanced through the guide catheter into the affected intracranial vessels, and angioplasty was performed as described below. In one case, additional IA nicardipine was administered through the lumen of a deflated Scepter XC balloon after angioplasty for the treatment of distal vasospasm that was not reachable with the Scepter balloon (the M3 segment of the middle cerebral artery).

\section{RESULTS}

Five consecutive patients (four women and one man) were identified who underwent endovascular vasospasm treatment that included cerebral angioplasty with the Scepter XC balloon. The average age of the patients was 50 years. Four patients presented with Fisher grade III-IV hemorrhage due to ruptured aneurysms arising from the anterior communicating artery, the A1 segment of the anterior cerebral artery, the basilar apex, or the middle cerebral artery trifurcation. Two of these aneurysms were treated by endovascular coiling and the other two aneurysms were treated by microsurgical clipping. A single patient presented with SAH following trans-clival resection of a skull base solitary fibrous tumor. These clinical characteristics are summarized in table 1.

All five patients developed drowsiness with or without focal neurologic deficits on post-hemorrhage days 5, 7, 8, or 14 that corresponded with cerebral vasospasm. Two patients required multiple endovascular treatment sessions that included angioplasty with the Scepter XC balloon for refractory vasospasm. The degree of vasospasm severity by cerebral angiography was severe in four patients and moderate in one patient. These data are summarized in table 2 .

A total of $10-60 \mathrm{mg}$ of IA nicardipine were administered prior to angioplasty in all patients. In the majority of cases (nine of 11 vasospasm treatment sessions), additional IA nicardipine
Table 1 Demographic and clinical details of vasospasm patients treated with Scepter balloons

\begin{tabular}{llllll}
\hline $\begin{array}{l}\text { Patient } \\
\text { No }\end{array}$ & $\begin{array}{l}\text { Fisher } \\
\text { grade }\end{array}$ & $\begin{array}{l}\text { Hunt- } \\
\text { Hess }\end{array}$ & $\begin{array}{l}\text { Aneurysm } \\
\text { location }\end{array}$ & $\begin{array}{l}\text { Aneurysm } \\
\text { treatment }\end{array}$ & Mortality \\
\hline 1 & III & II & Acom & Coil & No \\
2 & IV & III & A1 ACA & Clip & Yes \\
3 & N/A* & N/A* & N/A* & N/A* & No \\
4 & III & III & Basilar & Coil & No \\
5 & III & II & MCA & Clip & No \\
& & & trifurcation & & \\
\hline
\end{tabular}

*Subarachnoid hemorrhage and resulting vasospasm in patient No 3 was due to a complicated skull base tumor resection with significant postoperative hemorrhage. $\mathrm{ACA}$, anterior cerebral artery; Acom, anterior communicating artery; MCA, middle cerebral artery.

was administered through the guide catheter while cerebral angioplasty was performed using the Scepter XC balloon.

Multiple cerebral vessel segments were treated by balloon angioplasty with the Scepter XC balloon, including the supraclinoid ICA, the A1 and A2 segments of the anterior cerebral artery, the M1 and M2 segments of the middle cerebral artery, the V4 segment of the vertebral artery, and the basilar artery (see table 2). All angioplasty procedures resulted in a markedly improved caliber of the treated vessels with either minimal or no residual vasospasm identified. There was a corresponding clinical improvement in all patients (see example cases in figures 1-3).

In one patient, there was persistent vasospasm in the M3 segment of the middle cerebral artery that was deemed too distal for angioplasty with the Scepter XC balloon. In this case, the Scepter XC balloon was deflated and its position was maintained in the M1 segment of the middle cerebral artery after cerebral angioplasty. The guidewire was removed from the balloon, and additional IA nicardipine was administered through the central lumen of the deflated balloon directly into the M1 segment of the middle cerebral artery for targeted treatment of distal vasospasm. There was a marked improvement in the caliber of the M3 segment vessels following this additional nicardipine infusion.

There were no complications related to the angioplasty procedures. There was one mortality in the treated group that was secondary to a combination of increased intracranial pressure and stroke burden following aneurysm rupture and not directly related to endovascular vasospasm treatment.

\section{DISCUSSION}

Aggressive treatment of symptomatic cerebral vasospasm following SAH is essential in order to avoid vasospasm mediated cerebral infarction. Here we have described our initial experience with the extra compliant dual lumen Scepter XC balloon in the treatment of cerebral vasospasm following SAH.

The Scepter XC balloon performed well in the treatment of cerebral vasospasm in five consecutive patients. Patients undergoing cerebral angioplasty with the Scepter XC balloon demonstrated favorable angiographic improvement after treatment, and there were no procedure related complications in our series. The two principle assets of the Scepter XC balloon in the treatment of cerebral vasospasm are trackability and the extra compliant balloon design, both of which are discussed in more detail below.

We found the Scepter XC balloon to be extremely trackable, and we were able to position the balloon distally into the intracranial circulation (the A2 segment of the anterior cerebral 


\section{New devices}

Table 2 Vasospasm treatment details

\begin{tabular}{|c|c|c|c|c|c|}
\hline Patient No & Aneurysm location & Vasospasm severity & Days to vasospasm treatment & No of vasospasm treatments & Vessel segment angioplastied \\
\hline 1 & Acom & Severe & $7,8,9$ & 3 & ICA, A1, A2, M1 \\
\hline 2 & A1 ACA & Severe & $5,6,7,8,10$ & 5 & ICA, A1, A2, M1, M2, V4, basilar \\
\hline 3 & $\mathrm{~N} / \mathrm{A}$ & Severe & 8 & 1 & V4, basilar \\
\hline 4 & Basilar & Severe & 14 & 1 & V4, basilar \\
\hline 5 & MCA trifurcation & Moderate & 8 & 1 & M1, M2 \\
\hline
\end{tabular}

artery and M2 segment of the middle cerebral artery). The Scepter XC balloon accommodates a larger 0.014 inch microwire, which provides significant stability to the balloon during navigation of tortuous vessels and distal advancement. The superior steerability of a 0.014 inch microwire relative to the smaller 0.010 inch microwires that are used in single lumen balloons also facilitates successful navigation into the intracranial circulation. Lastly, we found that the 0.014 inch microwire provided increased stability to the balloon during inflation. This improved stability resulted in less slippage of the balloon along the course of the guidewire during inflation relative to the single lumen balloons that have been used in the past at our institution.

The extra compliant design of the Scepter XC balloon lends itself well to use in the treatment of cerebral vasospasm. In our experience during inflation, the balloon tended to conform to the course of the vessel concurrent with radial expansion. This conformation to the native vessel shape reflects the extra compliant nature of the balloon and, in our opinion, leads to a more controlled and gentle balloon inflation. Moreover, we found that the extra compliant design of the Scepter XC balloon facilitated angioplasty at branch points in the vessel by allowing the
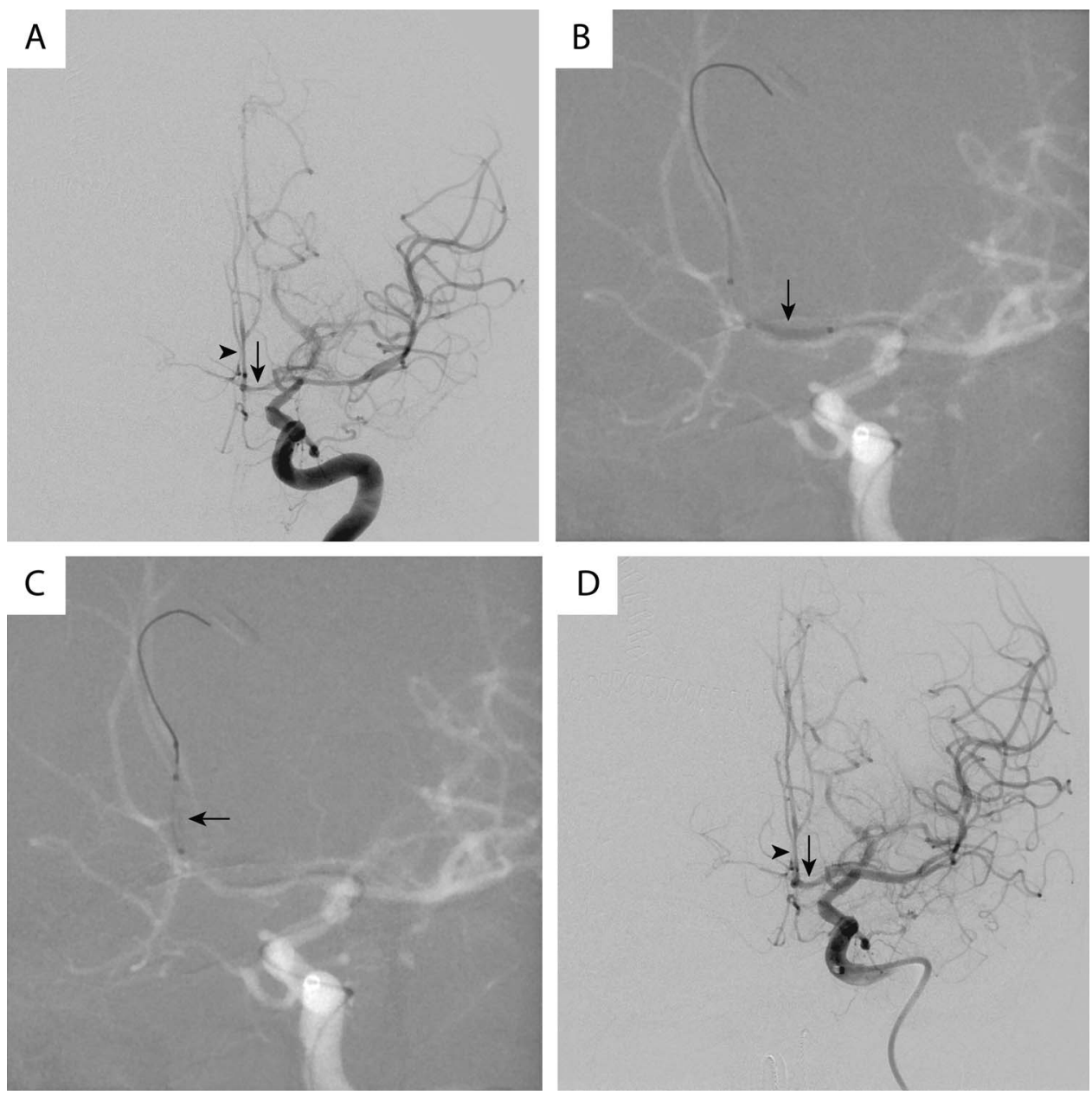

Figure 1 Cerebral angioplasty of the anterior cerebral artery (ACA) using the Scepter XC balloon. (A) Cerebral angiogram prior to endovascular vasospasm treatment demonstrating moderate vasospasm of the A1 (arrow) and A2 (arrowhead) segments of the left ACA. (B) Fluoroscopic image demonstrating angioplasty of the A1 segment of the ACA with the Scepter XC (arrow). A Traxcess microwire is noted more distally in the A2 and A3 segments of the left ACA. (C) Fluoroscopic image demonstrating angioplasty of the A2 segment of the left ACA with the Scepter XC (arrow). A Traxcess microwire is noted more distally in the A2 and A3 segments of the left ACA. (D) Cerebral angiogram following angioplasty of the left ACA with the Scepter XC balloon demonstrates an improved caliber of the A1 (arrow) and A2 (arrowhead) segments of the left ACA. 

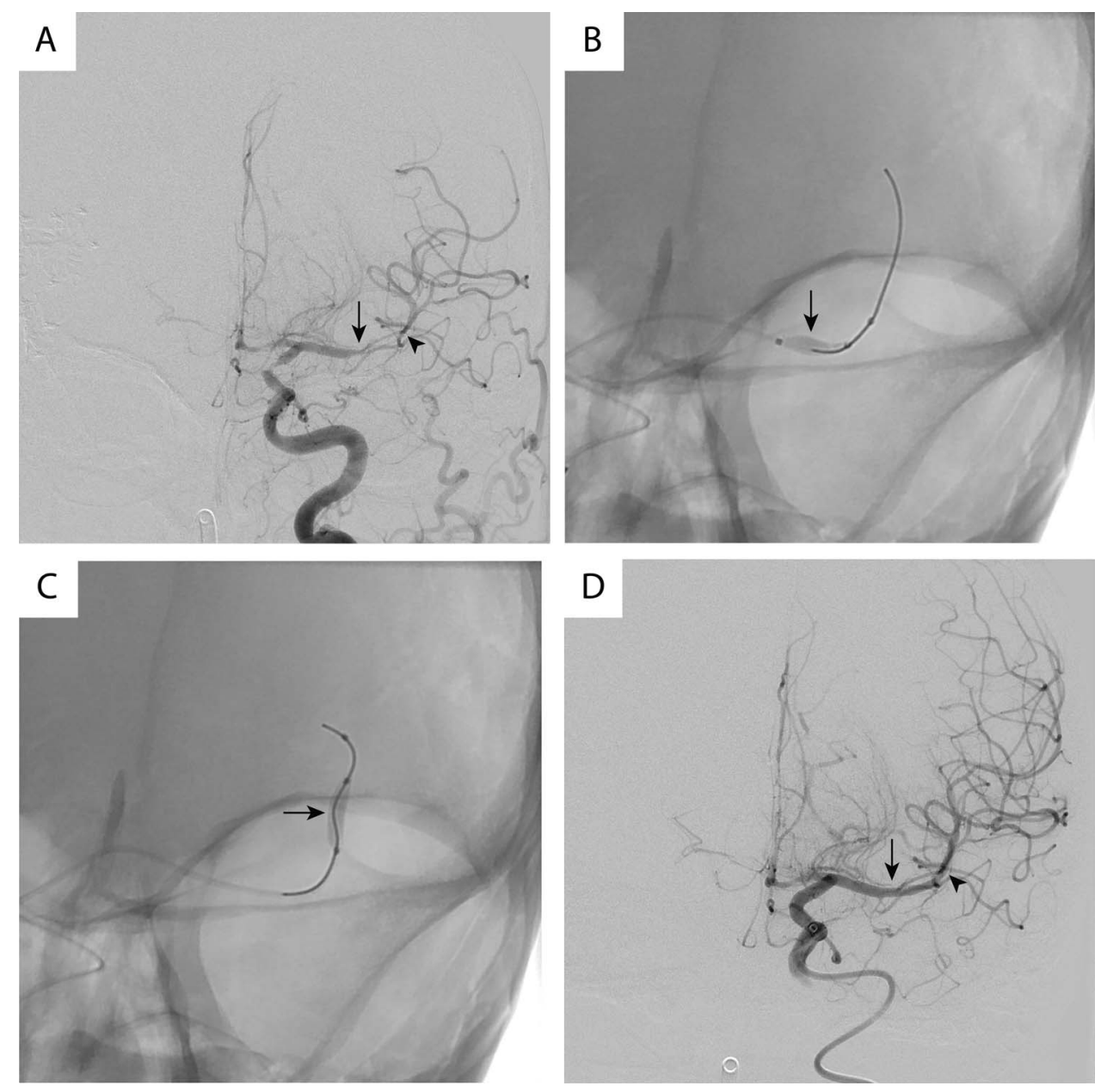

Figure 2 Cerebral angioplasty of the middle cerebral artery (MCA) using the Scepter XC balloon. (A) Cerebral angiogram prior to endovascular vasospasm treatment demonstrating severe vasospasm of the M1 (arrow) and M2 (arrowhead) segments of the left anterior cerebral artery (ACA). (B) Fluoroscopic image demonstrating angioplasty of the M1 segment of the ACA with the Scepter XC (arrow). A Traxcess microwire is noted more distally in the M2 segment of the left MCA. (C) Fluoroscopic image demonstrating angioplasty of the M2 segment of the left MCA with the Scepter XC (arrow). A Traxcess microwire is noted more distally in the M2 segment of the left MCA. (D) Cerebral angiogram following angioplasty of the left MCA with the Scepter XC balloon demonstrates an improved caliber of the M1 (arrow) and M2 (arrowhead) segments of the left MCA.

balloon to prolapse slightly into branching vessels, which resulted in increased stability during balloon inflation.

The trackability, stability, and extra compliant balloon design of the Scepter XC balloon facilitated safe and successful angioplasty of the intracranial circulation in our hands. We were able to successfully navigate the Scepter XC balloon into severely narrowed anterior and middle cerebral arteries. Furthermore, we successfully angioplastied very small vessels, including the A2 segment of the anterior cerebral artery and the M2 segment of the middle cerebral artery, without complication. However, we note that angioplasty of the cerebral vessels distal to these segments is unlikely to be prudent given the risk of vessel injury and rupture.

Careful planning prior to endovascular treatment of cerebral vasospasm can lead to decreased procedure time and maximal improvement in intracranial vessel narrowing. IA administration of nicardipine or other calcium channel blockers may augment the results of cerebral angioplasty. At our institution, we have developed a protocol in which IA nicardipine is infused through the guide catheter before and during cerebral angioplasty, which maximizes pharmacological vasodilation and circulating nicardipine levels and minimizes indwelling catheter time. Moreover, the ability to administer additional IA nicardipine through the lumen of the deflated Scepter XC balloon was beneficial in the targeted treatment of distal M3 vasospasm after more proximal vasospasm angioplasty in a single patient.

We experienced no procedure related complications in this small series although arterial injury or rupture is a concern in all cases of cerebral angioplasty. Previous studies have described a $1-4 \%$ risk of arterial rupture or dissection during angioplasty for the treatment of cerebral vasospasm, although these studies were performed with earlier generations of angioplasty balloons. ${ }^{11} 16$ Theoretically, the improved stability of balloon inflation and the extra compliant nature of the Scepter XC balloon should be protective against arterial injury and vessel rupture. It would be of interest to determine if the safety profile of cerebral angioplasty is improved with the Scepter XC balloon. However, much greater numbers of patients would be required to determine accurately the safety profile of the Scepter XC balloon in the treatment of cerebral vasospasm.

There are a few shortcomings to the Scepter XC balloon. First, the preparation of the balloon is somewhat time consuming in order to ensure an absence of air within the device. Next, the balloon has a $5 \mathrm{~mm}$ nose distal to the balloon that may limit positioning of the balloon near vessel bifurcations. Lastly, although not directly applicable to this study, the absence of a coil marker on the catheter portion of the balloon is limiting 

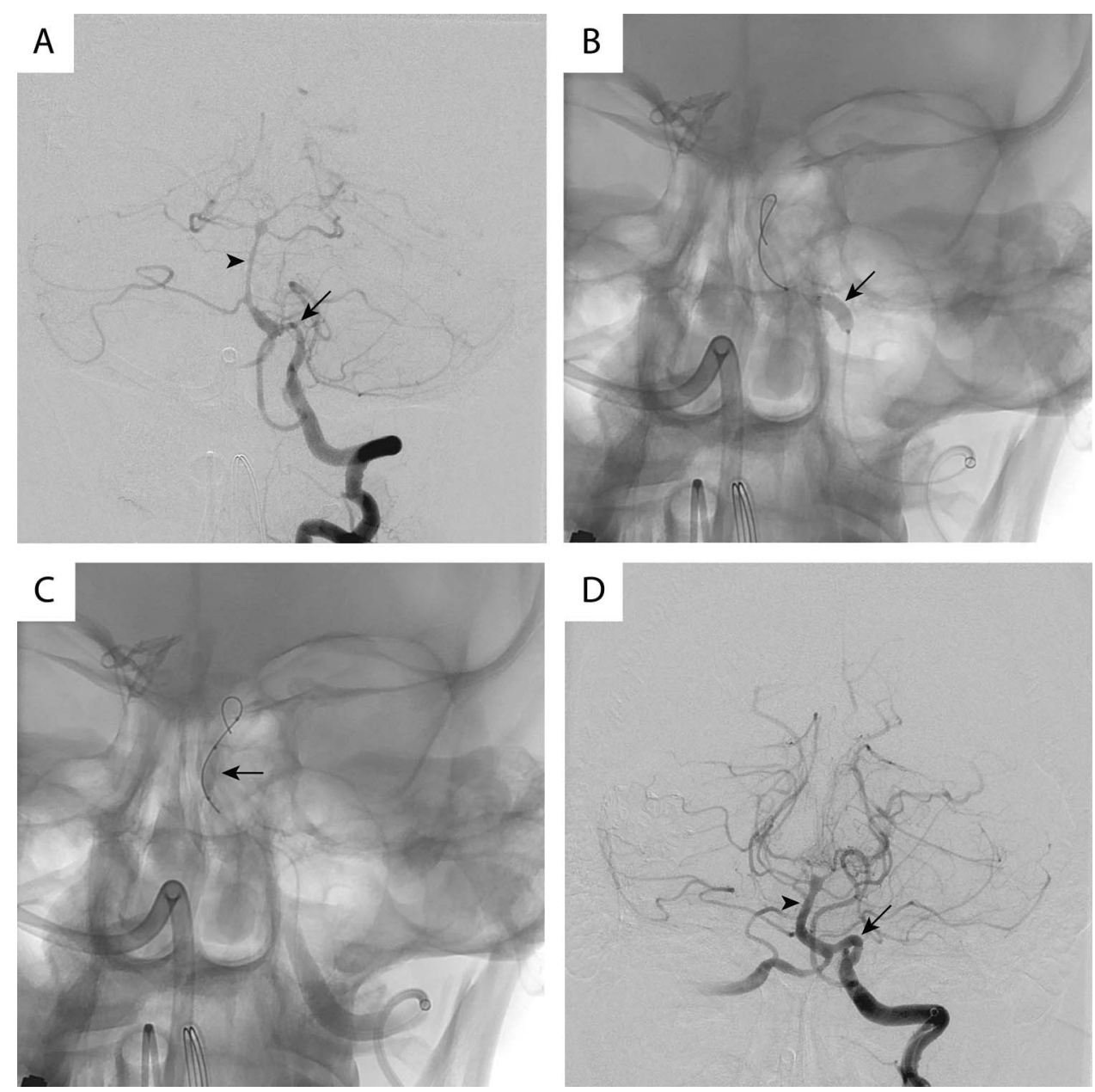

Figure 3 Cerebral angioplasty of the intradural vertebral artery (VA) and the basilar artery (BA) using the Scepter XC balloon. (A) Cerebral angiogram prior to endovascular vasospasm treatment demonstrating moderate to severe vasospasm of the V4 segment of the left VA (arrow) and severe vasospasm of the BA (arrowhead). (B) Fluoroscopic image demonstrating angioplasty of the V4 segment of the left VA with the Scepter XC (arrow). A Traxcess microwire is noted more distally in the BA. (C) Fluoroscopic image demonstrating angioplasty of the BA with the Scepter XC (arrow). A Traxcess microwire is noted more distally in the BA. (D) Cerebral angiogram following angioplasty of the V4 segment of the left VA and the BA with the Scepter XC balloon demonstrates an improved caliber of the V4 segment of the left VA (arrow) and the BA (arrowhead).

when using the Scepter XC in the treatment of cerebral aneurysms. Ideally, these limitations in the Scepter XC balloon will be improved with future generations of the device.

\section{CONCLUSIONS}

Cerebral angioplasty using the Scepter XC balloon in the treatment of vasospasm following $\mathrm{SAH}$ is safe and effective, and there were no complications in our series of five patients and 11 total angioplasty treatment sessions. The excellent trackability, stability, and the extra compliant balloon design of the Scepter XC facilitated successful angioplasty of distal intracranial vessels, including the A2 segment of the anterior cerebral artery and the M2 segment of the middle cerebral artery. Moreover, the ability to infuse IA calcium channel blockers through the central lumen of the Scepter XC provides an additional tool in the treatment of cerebral vasospasm.

Contributors $\mathrm{JJH}, \mathrm{OC}$, and HMD conceived and designed the study. JJH acquired the data and performed the data analysis. All authors were involved in the interpretation of the data, writing the manuscript, and revising the manuscript. All authors approved the final version of the manuscript for publication.

\section{Competing interests None.}

Ethics approval This study was performed in accordance with Stanford University's Internal Review Board policies and is in compliance with the Health Insurance Portability and Accountability Act.
Provenance and peer review Not commissioned; externally peer reviewed.

Data sharing statement All data are available within the manuscript. Additional data may be requested of the corresponding author if needed.

\section{REFERENCES}

1 Kassell NF, Torner JC, Jane JA, et al. The International Cooperative Study on the Timing of Aneurysm Surgery. Part 2: surgical results. J Neurosurg 1990;73:37-47

2 Kassell NF, Torner JC, Haley EC Jr, et al. The International Cooperative Study on the Timing of Aneurysm Surgery. Part 1: overall management results. J Neurosurg 1990;73:18-36.

3 Dorsch NW, King MT. A review of cerebral vasospasm in aneurysmal subarachnoid haemorrhage Part I: Incidence and effects. J Clin Neurosci 1994;1:19-26.

4 Kassell NF, Peerless SJ, Durward QJ, et al. Treatment of ischemic deficits from vasospasm with intravascular volume expansion and induced arterial hypertension. Neurosurgery 1982;11:337-43.

5 Wood JH, Simeone FA, Fink EA, et al. Hypervolemic hemodilution in experimental focal cerebral ischemia. Elevation of cardiac output, regional cortical blood flow, and ICP after intravascular volume expansion with low molecular weight dextran. J Neurosurg 1983;59:500-9.

6 Rosenwasser RH, Armonda RA, Thomas JE, et al. Therapeutic modalities for the management of cerebral vasospasm: timing of endovascular options. Neurosurgery 1999;44:975-9.

7 Zubkov YN, Nikiforov BM, Shustin VA. Balloon catheter technique for dilatation of constricted cerebral arteries after aneurysmal SAH. Acta Neurochir (Wien) 1984;70:65-79. 
8 Badjatia N, Topcuoglu MA, Pryor JC, et al. Preliminary experience with intra-arterial nicardipine as a treatment for cerebral vasospasm. AJNR Am J Neuroradiol 2004;25:819-26.

9 Biondi A, Ricciardi GK, Puybasset L, et al. Intra-arterial nimodipine for the treatment of symptomatic cerebral vasospasm after aneurysmal subarachnoid hemorrhage: preliminary results. AJNR Am J Neuroradio 2004;25:1067-76.

10 Feng L, Fitzsimmons BF, Young WL, et al. Intraarterially administered verapamil as adjunct therapy for cerebral vasospasm: safety and 2-year experience. AJNR Am J Neuroradiol 2002;23:1284-90.

11 Elliott JP, Newell DW, Lam DJ, et al. Comparison of balloon angioplasty and papaverine infusion for the treatment of vasospasm following aneurysmal subarachnoid hemorrhage. J Neurosurg 1998;88:277-84.
12 Brothers MF, Holgate RC. Intracranial angioplasty for treatment of vasospasm after subarachnoid hemorrhage: technique and modifications to improve branch access. AJNR Am J Neuroradiol 1990;11:239-47.

13 Higashida RT, Halbach VV, Dowd CF, et al. Intravascular balloon dilatation therapy for intracranial arterial vasospasm: patient selection, technique, and clinical results. Neurosurg Rev 1992;15:89-95.

14 Eskridge JM, Newell DW, Winn HR. Endovascular treatment of vasospasm. Neurosurg Clin N Am 1994:5:437-47.

15 Linskey ME, Horton JA, Rao GR, et al. Fatal rupture of the intracranial carotid artery during transluminal angioplasty for vasospasm induced by subarachnoid hemorrhage. Case report. J Neurosurg 1991;74:985-90.

16 Eskridge JM, McAuliffe W, Song JK, et al. Balloon angioplasty for the treatment of vasospasm: results of first 50 cases. Neurosurgery 1998;42:510-16. 